\title{
LOCAL SECTIONS OF FLOWS ON MANIFOLDS
}

\author{
WILLIAM C. CHEWNING AND R. STEVEN OWEN
}

ABSTRACT. We show the existence of local sections for dynamical systems on manifolds which are themselves generalized manifolds of codimension one. One implication of this is that the level surfaces of Lyapunov functions on three-manifolds are two-manifolds. This fact is used to study the geometry of stable attractors in three-manifolds.

A dynamical system is a triple $(X, R, \pi) ; X=$ any topological space, $R=$ reals, and $\pi$ is a continuous map: $X \times R \rightarrow X$, for every $x \in X, \pi(x, 0)=$ $x$ and $\pi\left(\pi\left(x, t_{1}\right), t_{2}\right)=\pi\left(x, t_{1}+t_{2}\right)$. Let $S \subset X$ and $T \subset R$; we denote $\pi(S \times T)$ as $S \pi T$ whether $S, T$ are sets or points. A local section $S \subset X$ of extent $\epsilon$ is any set such that $S \times(-\epsilon, \epsilon)$ is topologically imbedded in $X$ by $\pi$. For definitions of other concepts such as rest point, attractor, stable sets, etc. the reader is referred to [1].

Lemma 1. Let $X$ be a Tychonov space. If $p \in X$ is not a rest point for a dynamical system $(X, R, \pi)$, then there is a local section $S$ of extent $\epsilon>0$ such that $p \in S$ and $S \pi(-\epsilon, \epsilon)$ is open in $X$.

Proof. By [5, pp. 144-145] there is a local section $T$ of extent $2 \epsilon$ for $(X, R, \pi)$, containing $p$, such that $p$ is interior to $T \pi(-\epsilon, \epsilon)$. An open set $U$ about $p$ may be chosen such that $\bar{U}$ is interior to $T \pi(-\epsilon, \epsilon)$ and hence $\bar{U} \pi(-\epsilon, \epsilon)$ is interior to $T \pi(-2 \epsilon, 2 \epsilon)$. We set $S=T \cap U$; clearly $S$ is a local section of extent $2 \epsilon$.

If $S \pi(-\epsilon, \epsilon)$ is not open in $X$, there is a net $\left\{x_{\alpha}\right\}$ in $T \pi(-2 \epsilon, 2 \epsilon) \backslash S \pi(-\epsilon, \epsilon)$ which converges to $x \in S \pi(-\epsilon, \epsilon)$. Let $\tau: T \pi(-2 \epsilon, 2 \epsilon) \rightarrow(-2 \epsilon, 2 \epsilon)$ be the "time of return to $T$ " map; i.e., for $y \in T \pi(-2 \epsilon, 2 \epsilon), y \pi \tau(y) \in T$. Because $\tau$ is $\pi^{-1}$ followed by projection onto the second factor, followed by multiplication in $(-2 \epsilon, 2 \epsilon)$ by $-1, \tau$ is continuous.

Now $|\tau(x)|<\epsilon$, so without loss of generality, we assume also that $\left|\tau\left(x_{\alpha}\right)\right|<\epsilon$ for every $\alpha$. Thus $x_{\alpha} \epsilon(T \backslash S) \pi(-\epsilon, \epsilon)$ for every $\alpha$, so $x_{a} \pi \tau\left(x_{\alpha}\right) \epsilon$

Received by the editors October 17, 1973 and, in revised form, January 2, 1974 and February 14, 1974.

AMS (MOS) subject classifications (1970). Primary 34C 35, 34C 40, $54 \mathrm{H} 20$.

Key words and phrases. Local sections, stable attractors. 
$T \backslash S$ must converge to $x \pi \tau(x)$ in $S$. This is not possible, as $S$ is open relative to $T$. ***

We recall the concept of a generalized manifold due to Wilder [10]. In particular, classical Euclidean $n$-manifolds are $n$-dimensional generalized manifolds (n-g.m.'s). Conversely, noncompact, connected, metric k-g.m.'s are classical $k$-manifolds for $k=1,2$.

Theorem 1. Let $X$ be a Euclidean n-manifold, and $(X, R, \pi)$ a dynamical system on $X$. Then the set $S$ of Lemma 1 is an $(n-1)$-generalized manifold.

Proof. The set $S \pi(-\epsilon, \epsilon)$ is an open subset of an $n$-manifold, and therefore an $n$-manifold itself. The set $S \times(-\epsilon, \epsilon)$ is then an $n$-manifold. By Theorem 6 of [7], we may conclude that $S$ is itse lf a generalized manifold. Raymond proves, roughly, that $M \approx A \times B$ is a generalized $n$-manifold iff $A$ and $B$ are $k$ - and $r$-manifolds respectively, where $n=k+r$. **

Corollary 1. If $(X, R, \pi)$ is a dynamical system on an n-manifold $X$, and $K$ is any compact local section of extent $2 \epsilon>0$ in $X$, then $K$ lies in an $(n-1)-g . m . S$ which is a local section of extent $\epsilon$. Moreover, if $K$ is contained in an open set W, S may also be assumed to lie in $W$.

Proof. By results of Hájek [5, Pp. 144-145], a local section $T$ may be found, of extent $2 \epsilon$, such that $K \subset \operatorname{int}(T \pi(-\epsilon, \epsilon)) \subset W \pi(-\epsilon, \epsilon)$. The procedure of Lemma 1 may then be followed to produce $S$, which will be an $(n-1)$ g.m. ***

As is well known, a continuous flow on a 2-manifold has local sections which are 1-manifolds [5]. Though his terminology was different, it was known to Whitney [9] that for a nonrest point of a flow in $E^{3}$, local sections which were discs existed.

Corollary 2. If $(X, R, \pi)$ is a dynamical system on a 3-manifold $X$, and $T$ is a local section of extent $\epsilon>0$ containing a compact set $K$, and $K \subset$ int $T \pi(-\epsilon, \epsilon)$, then there is a neighborhood of $K$ in $T$ which is a 2-manifold.

Proof. The 2-g.m. $S$ that is obtained in this setting is a classical 2manifold [10]. **

Corollary 3. If $(X, R, \pi)$ is a dynamical system on a 3-manifold with boundary $\partial X$, and $p$ is a nonrest point of $\pi$, then there is a 2-cell $C$ which is a local section of extent $\epsilon>0, p \in C$, and $p$ is interior to $C \pi(-\epsilon, \epsilon)$.

Proof. If $p \notin \partial X$, then the conclusion follows from Corollary 2. We extend the dynamical system $\pi$ on $X$ to a system on $D(X)$, the double of $X$. 
Let $h_{1}: X \rightarrow X_{1}$ and $h_{2}: X \rightarrow X_{2}$ be homeomorphisms, and let $\pi_{1}$ and $\pi_{2}$ be the corresponding dynamical systems induced by $h_{1}$ and $h_{2}$ from $\pi$. Let $X_{1} \cup X_{2}$ denote the disjoint union of $X_{1}$ and $X_{2}$, and let $q$ : $X_{1} \cup X_{2} \rightarrow D(X)$ be the quotient map which identifies $p \in X_{1}$ and $q \in X_{2}$ iff there is an $x \in \partial X$ with $h_{1}(x)=p, h_{2}(x)=q . D(X)$, equipped with the quotient topology, is a 3-manifold without boundary.

We define $\tilde{\pi}: D(X) \times R \rightarrow D(X)$ by

$$
\tilde{\pi}(x, t)=\left\{\begin{array}{lllll}
q \circ \pi_{1}\left(q^{-1}(x), t\right) & \text { if } x \in & q\left(X_{1}\right) \\
q \circ \pi_{2}\left(q^{-1}(x), t\right) & \text { if } x \in & q\left(X_{2}\right)
\end{array}\right\} .
$$

Since these maps agree on $q\left(X_{1}\right) \cap q\left(X_{2}\right), \tilde{\pi}$ is a dynamical system. If $p \in \partial X$ is a nonrest point under $\pi$, then $p^{\prime}=q(p)$ is a nonrest point of $\pi$. Because $\partial X_{1}$ is an invariant 2-manifold under $\pi_{1}, q\left(\partial X_{1}\right)$ is an invariant 2 -manifold under $\pi$. Accordingly there is an arc $A$ in $q\left(\partial X_{1}\right)$ which contains $p^{\prime}$, is a section of extent $2 \epsilon>0$ in $q\left(\partial X_{1}\right)$, and such that $p^{\prime}$ is interior, in $q\left(\partial X_{1}\right)$, to $A \tilde{\pi}(-\epsilon, \epsilon)$.

Let $S$ be a 2-manifold local section of extent $\epsilon$ for $\tilde{\pi}$ in $D(X)$, such that $A \subset S$, and $A \subset$ int $S \pi(-\epsilon, \epsilon)$. In $S$, let $B$ be a small 2-cell about $p^{\prime}$ whose boundary intersects both components of $A \backslash\left\{p^{\prime}\right\}$. Now $A \cap \partial B$ contains points $r, s$, such that the subarc $\overparen{r s}$ of $A$ contains $p^{\prime}$ and the open arc $\overparen{r S}$ does not meet $\partial B$. Let $C^{\prime}$ denote the component of $B \backslash \widehat{r S}$ in $q\left(X_{1}\right)$. Then $\mathrm{cl}\left(C^{\prime}\right)$ is a 2 -cell in $q\left(X_{1}\right)$. We define $C=h_{1}^{-1} \circ q^{-1}\left(\operatorname{cl}\left(C^{\prime}\right)\right)$. **

Lyapunov functions. Let $(X, R, \pi)$ be a dynamical system. A set $K C$ $X$ is asymptotically stable if and only if there is a continuous function $F$, defined on an open subset containing $K$, with range in $[0, \infty)$ such that $F(x)=0$ exactly when $x \in K$, and for $x \in \operatorname{dom}(F) \backslash K$, and $t>0, F(x)>$ $F(x \pi t) . F$ is called a Lyapunov function for $K$. The level surfaces of $F$ are local sections of extent $+\infty$ for $\pi$, if $X$ is locally compact and $K$ is compact.

Theorem 2. Let $(X, R, \pi)$ be a dynamical system on an n-manifold $X$, and suppose that the compact set $K \subset X$ is asymptotically stable with Lyapunov function $F$. Then there is an $\epsilon>0$, such that for all $c, 0<c<\epsilon$, $F^{-1}(c)$ is a compact $(n-1)-g . m$. and a local section of extent $+\infty$.

Proof. Let us choose $\epsilon>0$ such that $F^{-1}[0, \epsilon]$ is a compact neighborhood of $K$. For fixed $c \in(0, \epsilon)$, choose $\delta$ such that $0<c-\delta<c+\delta<$ 
є. Then $F^{-1}(c)$ is a compact local section. Define $\sigma=\sup \left\{F(x \pi(\delta / 3)): x \in F^{-1}(c)\right\} \quad$ and $\quad \tau=\inf \left\{F(x \pi(-\delta / 3)): x \in F^{-1}(c)\right\}$. Then $\sigma<c<\tau, F^{-1}(\sigma, \tau) \subset F^{-1}(c) \pi(-\delta / 2, \delta / 2)$, and $F^{-1}(c)$ is interior to $F^{-1}(\sigma, \tau)$ because $F$ is continuous. Now by Corollary 1 , there is a local section $T$ of extent $\delta / 2$, with $F^{-1}(c) \subset T \subset F^{-1}(\sigma, \tau)$, and $T$ is an $(n-1)$ g.m. We claim that $F^{-1}(c)=T$. If $p \in T \backslash F^{-1}(c)$, then

$$
(p, 0) \in\left(T \backslash F^{-1}(c)\right) \times(-\delta / 2, \delta / 2)=T \times(-\delta / 2, \delta / 2) \backslash F^{-1}(c) \times(-\delta / 2, \delta / 2) .
$$

Because the restriction of $\pi$ to $T \times(-\delta / 2, \delta / 2)$ is injective,

$$
\left(T \backslash F^{-1}(c)\right)_{\pi}(-\delta / 2, \delta / 2)=T \pi(-\delta / 2, \delta / 2) \backslash F^{-1}(c)_{\pi}(-\delta / 2, \delta / 2) .
$$

Therefore $\pi(p, 0)=p \in T \subset T \pi(-\delta / 2, \delta / 2) \backslash F^{-1}(c) \pi(-\delta / 2, \delta / 2)$. This last inclusion is not possible because $T \subset F^{-1}(\sigma, \tau) \subset F^{-1}(c) \pi(-\delta / 2, \delta / 2)$.

Therefore $F^{-1}(c)$ is an $(n-1)$-g.m. **

Definition. Let $X$ be a subspace of a topological space $Y$. If there is a homeomorphism $h$ carrying $X \times(-1,1)$ onto an open neighborhood of $X$ in $Y$, such that $h(x, 0)=x$ for every $x \in X$, then $X$ is bicollared in $Y$.

Lemma 2. If $(X, R, \pi)$ is a dynamical system on an n-manifold $X$, and $K$ is a compact local section for $(X, R, \pi)$, then $K$ is contained in an $(n-1)$ g.m. local section $S$ which is bicollared in $X$.

Proof. In proving Corollary 1 one obtains a locally compact set $S$ such that $K \subset S$, for some $\epsilon>0, S$ is a local section of extent $\epsilon$, and $S_{\pi}(-\epsilon, \epsilon)$ is open in $X$. Therefore $\pi$ maps $S \times(-\epsilon, \epsilon)$ homeomorphically onto an open subset of $X$, so $S$ is bicollared. It is proven in Theorem 1 that $S$ is an $(n-1)-g . \mathrm{m} . * *$

From Lemma 2 and Theorem 2, we can prove the following:

Corollary 4. If $X$ in Theorem 2 is a 3-manifold, then $F^{-1}(c)$ is a compact bicollared 2-manifold in $X$. **

In what follows, we use Čech homology with integral coefficients.

Theorem 3. Let $X=S^{3}$ or $R^{3}$, and let $K$ be any compact, connected, nonseparating subset of $X$ with $H_{1}(K)=0$. If $K$ is asymptotically stable for $(X, R, \pi)$, then any Lyapunov function for $K$ has level surfaces which are 2-spheres, bicollared in $X$.

Proof. Let $A(K)$ denote the region of attraction of $K . A(K) \backslash K$ is connected, for if $A(K) \backslash K$ were separated, then $X \backslash K$ would also be separated 
because $K$ is interior (in $X$ ) to $A(K)$. Let $F$ be a Lyapunov function for $K$ defined on $A(K)$. If $c>0$ is a value of $F$, then $F^{-1}(c)$ is a section for the parallelizable flow $\pi$ on $A(K) \backslash K$ [1, Exercise 3.14.2, p. 83]. Because $A(K) \backslash K$ is connected, $F^{-1}(c)$ must be connected $[1$, Lemma 2.3, p. 48].

Therefore $F^{-1}(c)$ is a compact, connected, bicollared 2-manifold imbedded in $S . F^{-1}(c)$ is either a sphere, a connected sum of tori, or a connected sum of projective planes $[6$, Theorem $5.1, \mathrm{p} .10]$. Set $B=F^{-1}[0, c]$, and for each positive integer $n$, set $B_{n}=B \pi n$. Then $B_{n} \subset B_{n-1}, B_{i}$ is homeomorphic to $B_{j}$ for all positive integers $i, j$, and $K=\bigcap_{n=1}^{\infty} B_{n}$. By the continuity of Čech homology, $H_{1}(K) \approx H_{1}(B)$. If $F^{-1}(c)=\partial B$ were a connected sum of tori, then $H_{1}(B)$ would be nontrivial. If $F^{-1}(c)$ were a connected sum of projective planes, then it could not be a compact subset of $X[6, \mathrm{p} .51]$. Therefore $F^{-1}(c)$ is a 2 -sphere. **

Theorem 4. Under the hypothesis of Theorem 3, the following hold:

(1) $A(K)$, the region of attraction for $K$, is homeomorphic to an open 3-cell.

(2) $K$ contains a rest point.

(3) There is a dynamical system $\bar{\pi}$ on $X$ with a rest point $p$, such that a homeomorphism exists from $X \backslash K$ onto $X \backslash\{p\}$ which takes sensed orbits of $\pi$ onto sensed orbits of $\bar{\pi}$.

Proof. To prove (1), let $c$ be such that $F^{-1}(c)$ is a bicollared 2-sphere, so that $B \equiv F^{-1}[0, c)$ is an open 3-cell [2a]. Then, as is easily seen, $A(K)=\bigcup_{n=1}^{\infty} B \pi(-n), B \pi(-n) \subset B \pi(-n-1)$, so $A(K)$ is a monotone union of open 3-cells, hence an open 3-cell [2b].

To prove (2), we note that $F^{-1}[0, c]$ is a closed 3 -cell which is invariant under the flow. Hence it must contain a rest point $[1$, p. 82$]$ which is clearly in $K$.

Finally, we observe that $K$ is cellular, i.e. $K=\bigcap_{n=1}^{\infty} C_{n}$, where $C_{n} \subset$ int $C_{n-1}$ and each $C_{n}$ is a 3-cell. To see this, just set $C_{n}=B \pi n$. Therefore, there is a continuous function $h: X \rightarrow X$ which takes $K$ to the point $p$ and such that $h: X \backslash K \rightarrow X \backslash\{p\}$ is a homeomorphism [7].

We define a dynamical system $(X, R, \bar{\pi})$ by

$$
\bar{\pi}(x, t)=\left\{\begin{array}{l}
p, \quad \text { if } x=p \\
b \circ \pi\left(b^{-1}(x), t\right), \quad \text { if } x \neq p
\end{array}\right\} .
$$

It is easy to check that $\bar{\pi}$ satisfies the conditions of (3).**

The authors thank Peter W. Harley for suggesting this analogue of Theorem 4 . 
Theorem 5. If $K \subset R^{n}$ is cellular and is asymptotically stable under $\left(R^{n}, R, \pi\right)$, then:

(1) $A(K)$ is homeomorphic to an open n-cell.

(2) $K$ contains a rest point.

(3) There is a dynamical system $\bar{\pi}$ on $R^{n}$ with rest point $p$, such that a homeomorphism exists from $R^{n} \backslash K$ onto $R^{n} \backslash\{p\}$ which takes sensed orbits of $\pi$ onto sensed orbits of $\bar{\pi}$.

Proof. Because $K$ is cellular, there is an open $n$-cell $B$, with $K \subset B C$ $A(K)$. We define, for each positive integer $n, B_{n}=B \pi(-n)$. Then $B_{n} C$ int $B_{n+1}$ and $A(K)=\bigcup_{n=1}^{\infty} B_{n}$. Therefore $A(K)$ is homeomorphic to an open $n$-cell [2b].

The arguments for (2) and (3) are similar to those given for Theorem 4. **

Theorem 6. If $X=S^{3}$ or $R^{3}$, and $(X, R, \pi)$ is a dynamical system for which $K$ is an asymptotically stable continuum with $H_{1}(K) \approx Z$, then the level surfaces of a Lyapunov function for $K$ are tori, bicollared in $X$.

Proof. The proof of Theorem 3 can be slightly modified to yield this result. **

An imbedding $f: P \rightarrow Q$ of a polyhedron $P$ into a polyhedron $Q$ is tame if there is a homeomorphism $h: Q \rightarrow Q$ such that $h \circ f$ is a piecewise linear imbedding. For example, a simple closed curve in $R^{3}$ or $S^{3}$ is tame if there is a space homeomorphism which takes the simple closed curve onto a polyhedral simple closed curve.

Corollary. Suppose, in Theorem $6, K$ is a nontrivial periodic orbit. Then, as a simple closed curve, $K$ is tamely imbedded in $X$.

Proof. We say that $K$ has the concentric enclosure property if $K=$ $\bigcap_{n=1}^{\infty} T_{n}$, where each $T_{n}$ is a solid torus, with $T_{n} \subset T_{n-1}$ and $\operatorname{cl}\left(T_{n-1} \backslash T_{n}\right)$ is homeomorphic to the product of a torus and an interval. If we set $T=F^{-1}[0, c]$ and $T_{n}=T \pi n$, then $K=\bigcap_{n=1}^{\infty} T_{n}$.

It is not hard to show that $\operatorname{cl}\left(T_{n} \backslash T_{n+1}\right)$ is homeomorhpic to a torus crossed with an interval. Let $M=F^{-1}(c) ; M$ is a torus and $M$ is the boundary of $T$. Furthermore $M \pi m$ is the boundary of $T \pi m$, so $M \pi[n-1, n) \approx T_{n-1} \backslash T_{n}$ so $\mathrm{cl}\left[T_{n-1} \backslash T_{n}\right] \approx M \times[n-1, n]$.

Therefore $K$ has the concentric enclosure property. Additionally, at each point $p$ of $K$, there is a local section $S$ which can be chosen to be a 2-cell, and which meets $K$ only in $p$. Under these conditions, $K$ is tamely imbedded in $X$ [4]. ** 


\section{REFERENCES}

1. N. P. Bhatia and G. P. Szegö, Stability theory of dynamical systems, Die Grundlehren der math. Wissenschaften, Band 161, Springer-Verlag, New York and Berlin, 1970. MR 44 \# 7077.

2a. M. Brown, A proof of the generalized Schoenflies theorem, Bull. Amer. Math. Soc. 66 (1960), 74-76. MR 22 \# 8470b.

2b. - The monotone union of open $n$-cells is an open $n$-cell, Proc. Amer. Math. Soc. 12 (1961), 812-814. MR 23 \# A4129.

3. W. C. Chewning, A dynamical system on $E^{4}$ neither isomorphic nor equivalent to a differential system, Bull. Amer. Math. Soc. 80 (1974), 150-153.

4. C. H. Edwards, A characterization of tame curves in the 3-sphere, Notices Amer. Math. Soc. 7 (1960), 875. Abstract \# 573-32.

5. O. Hájek, Dynamical systems in the plane, Academic Press, New York, 1968. MR 39 \# 1767.

6. W. S. Massey, Algebraic topology: An introduction, Harcourt, Brace, and World, New York, 1967. MR 35 \#2271.

7. F. A. Raymond, Separation and union theorems for generalized manifolds with boundary, Michigan Math. J. 7 (1960), 7-21. MR 22 \# 11388.

8. T. B. Rushing, Topological embeddings, Academic Press, New York, 1973.

9. H. Whitney, Cross sections of curves in 3-space, Duke Math. J. 4 (1938), $222-226$.

10. R. L. Wilder, Topology of manifolds, Amer. Math. Soc. Colloq. Publ., vol. 32, Amer. Math. Soc., Providence, R. I., 1949. MR 10, 614.

DEPARTMENT OF MATHEMATICS, UNIVERSITY OF SOUTH CAROLINA, COLUMBIA, COLUMBIA, SOUTH CAROLINA 29208 (Current address of W. C. Chewning)

DEPARTMENT OF MATHEMATICS, NAVAL POSTGRADUATE SCHOOL, MONTEREY, CALIFORNIA 93940

Current address(R. S. Owen): U. S. S. Independence, Norfolk, Virginia 